\title{
Estudo Comparativo de Áreas Endêmicas de Filariose Bancroftiana na Região Metropolitana do Recife, Brasil
}

\author{
Comparative Studies on Endemic Areas of Bancroftian Filariasis in \\ Greater Recife, Brazil
}

\author{
Maria Amélia V. Maciel ${ }^{1}$; Keyla Belizia F. Marzochi' ${ }^{2}$ Edite C. Silva ${ }^{1}$; \\ Abraham Rocha ${ }^{1} \&$ André F. Furtado ${ }^{1}$
}

\begin{abstract}
MACIEL, M. A. V.; MARZOCHI, K. B. F.; SILVA, E. C.; ROCHA, A. E FURTADO, A. F. Comparative Studies on Endemic Areas of Bancroftian Filariasis in Greater Recife, Brazil. Cad. Saúde Públ., Rio de Faneiro, 10 (supplement 2): 301-309, 1994.

Two districts in Recife (Santo Amaro and Campo Grande) and two districts in Olinda (Sapucaia and Salgadinho), were selected for a comparative study of bancroftian filariasis in Greater Recife. Selection parameters included similar socio-economic, demographic, and endemic levels of lymphatic filariasis. In the districts studied, streets were chosen randomly. These clusters consisted of 110 people each. A population sample was stratified by sex and age: 0-4, 5-9, 10-14, 15-19, 20-29, 30-39, 40-49, 50-59 and 60 years old and over. The parasitological data were obtained by measuring thick blood smears (60 $\mathrm{ul}$ ), collected from 8:00 to 12:00 PM and processed and stained with hematoxylin. The data were described in tables, and logarithimic expression graphics were used to analyze parasitic densities. In general, Recife showed a prevalence of 13.5\%, and Olinda 12.3\%. Mean parasitic densities were 41 and 70 microfilariae $/ 60 \mu \mathrm{l}(\mathrm{mf} / 60 \mu \mathrm{l})$, respectively. Higher microfilaremic rates were observed in Recife for the 20-29-year age bracket and in Olinda in the 30-39-year bracket. The authors concluded that the disease has returned to former endemic levels, and the data described call for a reevaluation of control campaigns carried out by the Brazilian National Health Foundation, since the endemic has already reached serious proportions.
\end{abstract}

Key words: Filariasis; Wuchereria bancrofti; Epidemiology

\section{INTRODUÇÃO}

A filariose corresponde a um grupo de doenças associadas a helmintos do filo Nematoda, superfamília Filarioidea. No Brasil encontram se as espécies Wuchereria, Onchocerca, e

${ }^{1}$ Este estudo recebeu apoio financeiro do UNPD/World Bank/WHO Special Programme for Research and Training in Tropical Diseases (TDR); Conselho Nacional de Desenvolvimento Científico e Tecnológico (CNPq) e Fundação de Amparo à Ciência e Tecnologia do Estado de Pernambuco (Facepe).

${ }^{1}$ Centro de Pesquisas Aggeu Magalhães da Fundação Oswaldo Cruz. Avenida Moraes Rego, s/n, Campus da Universidade Federal de Pernambuco, Recide, PE, 50670 420, Brasil.

${ }^{2}$ Escola Nacional de Saúde Pública da Fundação Oswaldo Cruz. Rua Leopoldo Bulhões, 1480, Rio de Faneiro, RF, 21041-210, Brasil.
Mansonella, causando variado espectro clinicopatológico. A denominação "filariose linfática" está relacionada a três parasitos, Wuchereria bancrofti, Brugia timori, e Brugia malayi.

Embora a filariose bancroftiana possa ocasionar considerável grau de morbidade, relacionado às formas agudas e crônicas da patologia, a mortalidade é quase nula.

Segundo estimativa da Organização Mundial da Saúde (1992), três bilhões de pessoas vivem em países endêmicos, 75 milhões estão infectadas, entre as quais, 72 milhões por $W$. bancrofti. A distribuição geográfica da infecção/doença pela $W$. bancrofti atinge países como China, Indonésia, Índia e países africanos (WHO, 1992).

A filariose teria chegado ao Brasil pelo tráfico de escravos; entretanto, os primeiros 
estudos sobre a doença só vieram a ser efetuados na década de 1940 (Parahym, 1961). O maior número de pesquisas sobre a endemia foi realizado por Rachou que, entre 1952 e 1957, implementou inquéritos epidemiológicos, identificando focos autóctones em várias cidades, de norte a sul do Brasil (Rachou, 1954, 1957a, 1957b; Rachou et al., 1952, 1957). Focos endêmicos de filariose permanecem nas cidades de Recife, Belém e Maceió.

De 1954 a 1985, o Serviço Nacional de Campanhas de Filariose da Superitendência de Campanhas de Saúde Pública, atualmente Fundação Nacional de Saúde(FNS), realizou vários inquéritos epidemiológicos, incluindo o Recife, afirmando os boletins de 1985 que a endemia estaria controlada no Brasil, pois apresentava índices considerados de "baixa endemicidade" (MS, 1985). Para toda a cidade do Recife a taxa era de 1,5\% (MS, 1985). Entretanto, pesquisas posteriores feitas pelo Centro de Pesquisas Aggeu Magalhães/Fiocruz evidenciaram que a endemia continua a ser um sério problema de saúde pública, havendo grande número de indivíduos expostos, infectados e doentes, com considerável aumento endêmico em extensão e intensidade, e, portanto, número crescente tanto de portadores de microfilaremia como de pessoas com formas agudas e crônicas na área metropolitana (Dreyer, 1987).

Admitimos a hipótese de que, em áreas endêmicas de filariose, correspondentes a regiões metropolitanas do Recife, possa haver diferenças no comportamento da infecção/doença devido não só a pecularidades na ocupação de espaço pelas populações, mas, principalmente, em relação às medidas pouco eficazes de controle, historicamente efetuadas em diversas áreas, que, de comum, possuem apenas o baixo padrão sócio-econômico. Não há informações precisas sobre a forma de ocupação das áreas metropolitanas do Recife em relação à procedência das populações, embora a instalação mais recente dessas comunidades date de, aproximadamente, duas décadas, enquanto a ocupação antiga remonta a cerca de cinco décadas. Nestas últimas, o programa de controle da filariose vem sendo realizado pela FNS (Superintendência de Campanhas de Saúde Pública - Sucam) desde os anos 50, por meio de identificação e tratamento dos casos positi- vos e combate ao vetor utilizando inseticidas, enquanto nas áreas de ocupação mais recente nenhum programa de controle foi iniciado até o momento.

Pretendemos, assim, verificar as possíveis diferenças entre conglomerados urbanos de instalação antiga e instalação recente, onde o combate à endemia vem ocorrendo há décadas ou apenas recentemente, respectivamente, considerando a distribuição da filariose conforme densidade parasitária humana e ocorrência por grupo etário e sexo.

\section{MATERIAL E MÉTODOS}

Para o estudo comparativo foram selecionados dois bairros na cidade do Recife, Santo Amaro e Campo Grande, e dois bairros na cidade de Olinda, Sapucaia e Salgadinho, cidades que compõem a Região Metropolitana do Recife.

O método epidemiológico aplicado para realizar este estudo foi a técnica de amostragem por conglomerados. Dos bairros selecionados, foram sorteadas três ruas, e, nelas, um ponto inicial, a partir do qual se formavam os conglomerados, que poderiam ser compostos de uma rua ou mais.

A razão pela qual os referidos bairros das duas cidades foram selecionados para o estudo foi a diferença de tempo de instalação da endemia entre eles. As áreas consideradas da cidade do Recife têm historicidade endêmica de filariose de quase cinco décadas; a instalação de suas comunidades remonta ao ano de 1940. Já nas áreas de Olinda, ocupadas por volta da década de 1970, a verificação da parasitose remonta aos anos 80 (Dreyer, 1987).

Em cada um dos bairros foram verificadas as taxas de endemicidade de filariose bancroftiana e as respectivas características sócio-econômicas, tais como habitação (tipo, número de cômodos e de residentes), renda familiar e condições de saneamento. Esses dados foram obtidos de todas as famílias das áreas amostrais, objetivando a definição de estratos populacionais com padrão sócio-econômicos similares nas diferentes áreas, para comparação.

As amostras populacionais foram distribuídas por sexo, idade, endereço e tempo de moradia na área, sendo excluídos da análise os morado- 
res com menos de um ano de residência e as crianças abaixo de cinco anos, uma vez que a hipótese considerava que o pouco tempo de exposição à infecção não justificaria o exame desses grupos (OMS, 1988).

Os dados parasitológicos foram obtidos mediante o exame de gota espessa mensurada, $60 \mu \mathrm{l}$ de sangue, coletada entre $20 \mathrm{~h}$ e $24 \mathrm{~h}$. As lâminas foram processadas e coradas pelo método de Carrazi com rígido controle de qualidade, $20 \%$ das lâminas positivas ou negativas foram reaxaminadas.

As variáveis, sexo, idade, microfilaremia e densidade parasitária, estão expressas em tabelas e, na análise estatística, utilizou-se o teste de qui-quadrado.

\section{RESULTADOS}

Em Recife, as áreas estudadas constituíram-se de quatro conglomerados, tendo cada um 110 pessoas em média. Foram examinadas, no total, 466 pessoas, cuja idade variou entre 03 e 68 anos, estando $42 \%$ da amostra populacional entre 03 e 19 anos; 45,7\% eram do sexo masculino, e 54,3\%, do sexo feminino. A média de habitantes por residência foi de cinco pessoas, com variação de uma a 18 .

A área estudada em Olinda constituiu-se de seis conglomerados, com 110 pessoas em média. Foram examinadas, ao todo, 685 pessoas, cuja idade variou de 03 a 78 anos, dos quais $43 \%$ correspondiam a indivíduos entre 03 e 19 anos; $45,5 \%$ pertenciam ao sexo masculino, e $54,5 \%$, ao sexo feminino. A média de residentes por moradia foi de quatro pessoas, com variação de uma a 20 .

A drenagem das águas, na área de Recife, é feita para canais localizados a cerca de 100 metros dos conglomerados; $85 \%$ das residências geralmente possuem fossas sem suspiros, como forma de saneamento.

Em Olinda, 87\% das casas possuem fossas com suspiro, e os dejetos escoam a céu aberto, para canaletas em frente às residências. Em ambas as áreas, 95\% das casas são de alvenaria, com dois quartos em média.

Quanto às taxas de infecção por $W$. bancrofti, em Recife, das 466 pessoas examinadas, 63 $(13,5 \%)$ eram positivas, enquanto, em Olinda, entre as 685 pessoas examinadas, 84 (12,3\%) apresentaram exames positivos.

A distribuição por faixa etária e sexo demonstrou que, em ambas as áreas, a faixa etária de 5-9 anos apresenta os menores índices (Tabelas 1 e 2).

A Tabela 1 das áreas do Recife mostra que, em ambos sexos, as menores taxas, abaixo de $10 \%$, estão nos menores de 9 anos; há consideravél elevação a partir dos 10 até 49 anos, cujas taxas estão acima daquele patamar, com médias respectivas de $17 \%$ e $14 \%$ para os sexos masculino e feminino; acima dos 50 anos, a distribuição foi irregular, com queda em maio-

TABELA 1. Distribuição de Filariose Bancroftiana por Sexo e Idade nos Conglomerados na Área Endêmica do Recife (Santo Amaro e Campo Grande), no Ano de 1991

\begin{tabular}{|c|c|c|c|c|c|c|}
\hline \multirow[b]{2}{*}{$\begin{array}{l}\text { Faixa Etária } \\
\text { (anos) }\end{array}$} & \multicolumn{2}{|c|}{$\mathrm{N}^{\circ}$ Examinados } & \multicolumn{4}{|c|}{$\mathrm{N}^{\circ}$ Positivos } \\
\hline & $\mathrm{M}$ & $\mathrm{F}$ & $\mathrm{M}$ & $\%$ & $\mathrm{~F}$ & $\%$ \\
\hline $0-4$ & 1 & 1 & - & - & - & \\
\hline $5-9$ & 39 & 32 & 2 & 5,13 & 3 & 9,38 \\
\hline $10-14$ & 43 & 26 & 6 & 13,95 & 4 & 15,38 \\
\hline $15-19$ & 21 & 39 & 5 & 23,81 & 4 & 10,26 \\
\hline $20-29$ & 41 & 45 & 9 & 21,95 & 7 & 15,56 \\
\hline $30-39$ & 27 & 36 & 4 & 14,81 & 6 & 16,67 \\
\hline $40-49$ & 19 & 32 & 2 & 10,53 & 4 & 12,50 \\
\hline $50-59$ & 11 & 20 & 2 & 18,18 & - & \\
\hline $60 e+$ & 11 & 22 & 1 & 9,09 & 4 & 18,18 \\
\hline Total & 213 & 253 & 31 & 14,55 & 32 & 12,65 \\
\hline
\end{tabular}

$$
M=\text { Masculino } \quad F=\text { Feminino } \quad-=\text { Casos Negativos }
$$


TABELA 2. Distribuição de Filariose Bancroftiana por Sexo e Idade nos Conglomerados na Área Endêmica de Olinda (Salgadinho e Sapucaia), no Ano de 1991

\begin{tabular}{lccrrrr}
\hline \hline \multirow{2}{*}{$\begin{array}{l}\text { Faixa Etária } \\
\text { (anos) }\end{array}$} & \multicolumn{2}{c}{$\mathrm{N}^{\circ}$ Examinados } & \multicolumn{5}{c}{$\mathrm{N}^{\circ}$ Positivos } \\
& \multicolumn{1}{c}{$\mathrm{M}$} & $\mathrm{F}$ & $\mathrm{M}$ & $\%$ & $\mathrm{~F}$ & $\%$ \\
\cline { 2 - 7 } & 6 & 5 & - & - & - & \\
\hline $0-4$ & 49 & 45 & 2 & 4,08 & 2 & 4,44 \\
$5-9$ & 57 & 52 & 12 & 21,05 & 3 & 5,77 \\
$10-14$ & 38 & 47 & 3 & 7,89 & 7 & 14,89 \\
$15-19$ & 61 & 73 & 13 & 21,31 & 4 & 5,48 \\
$20-29$ & 44 & 56 & 10 & 22,73 & 8 & 14,29 \\
$30-39$ & 24 & 37 & 4 & 16,67 & 5 & 13,51 \\
$40-49$ & 15 & 22 & 4 & 26,67 & 2 & 9,09 \\
$50-59$ & 17 & 37 & 1 & 5,88 & 4 & 10,81 \\
$60 \mathrm{e}+$ & 311 & 374 & 49 & 15,76 & 35 & 9,36 \\
\hline Total & & & & & & \\
\hline \hline
\end{tabular}

$$
M=\text { Masculino } \quad \mathrm{F}=\text { Feminino } \quad-=\text { Casos Negativos }
$$

res de 60 anos do sexo masculino. No total, a positividade foi mais baixa no sexo feminino, porém sem diferença significativa $(\mathrm{p}>0,05)$.

A Tabela 2, referente às áreas de Olinda, indica, também as menores taxas de microfilaremia em menores de 10 anos; acima dessa idade, porém, a freqüência é bastante irregular e não obedece a um padrão, sendo em média de 19,4 e $10,5 \%$ para o sexo masculino e feminino, respectivamente; há nítida tendência à queda no sexo masculino, em maiores de 60 anos. Do total de positivos, a freqüência de microfilarêmicos foi menor no sexo feminino, com diferença significativa $(\mathrm{p}<0,03)$.

Em Recife, a análise estratificada por faixa etária demonstrou que o maior índice de percentagem de microfilarêmicos se situa entre 20-29 anos, enquanto o de Olinda fica entre 30-39 anos (Tabela 3, Figura 3).

Quando comparamos idade, sexo e índices microfilarêmicos em Recife, ambos os sexos, na faixa etária de 10-39 anos, apresentam índices elevados, que declinam a partir dos 60 anos no sexo masculino, enquanto, no sexo feminino, 0 declínio ocorre antes, na faixa de 40-59 anos (Figura 1).

Em Olinda, nas faixas etárias de 10 a 39 anos também encontramos índices elevados, que declinam a partir dos 40 anos em ambos os sexos, com exceção da faixa etária de 50-59
TABELA 3. Distribuição de Filariose Bancroftiana por Faixa Etária nos Conglomerados nas Áreas Endêmicas do Recife e Olinda, no Ano de 1991

\begin{tabular}{lrrrr}
\hline \hline Faixa & \multicolumn{2}{c}{$\mathrm{N}^{\mathrm{o}}$ Positivos } & \multicolumn{2}{c}{$\mathrm{N}^{\mathrm{o}}$ Positivos } \\
\cline { 2 - 5 } $\begin{array}{l}\text { Etária } \\
\text { (anos) }\end{array}$ & Recife & \multicolumn{1}{c}{$\%$} & Olinda & $\%$ \\
\hline $0-4$ & - & - & - & - \\
$5-9$ & 5 & 5,60 & 4 & 4,2 \\
$10-14$ & 10 & 14,60 & 15 & 13,3 \\
$15-19$ & 9 & 16,60 & 10 & 11,3 \\
$20-29$ & 16 & 18,70 & 17 & 13,3 \\
$30-39$ & 10 & 15,70 & 18 & 18,4 \\
$40-49$ & 6 & 9,20 & 9 & 15,0 \\
$50-59$ & 2 & 9,40 & 6 & 17,8 \\
$60 \mathrm{e}+$ & 5 & 11,30 & 5 & 8,3 \\
\hline Total & 63 & 13,52 & 84 & 12,26 \\
\hline \hline
\end{tabular}

$-=$ Casos Negativos

anos (Figura 2). As Tabelas 4 e 5 apresentam a distribuição da freqüência de casos de microfilarêmicos por $60 \mu \mathrm{l}$ de sangue em Recife e Olinda. Recife apresentou valor médio de densidade microfilarêmica de $41 \mathrm{mf} / 60 \mu \mathrm{l}$ sangue, enquanto, em Olinda, esse valor foi de 70 microfilárias/60 $\mu 1$ (Tabelas 4 e 5). 
FIGURA 1. Distribuição do Índice Microfilarêmico por Sexo e Faixa Etária nos Conglomerados na Área Endêmica do Recife, no Ano de 1991

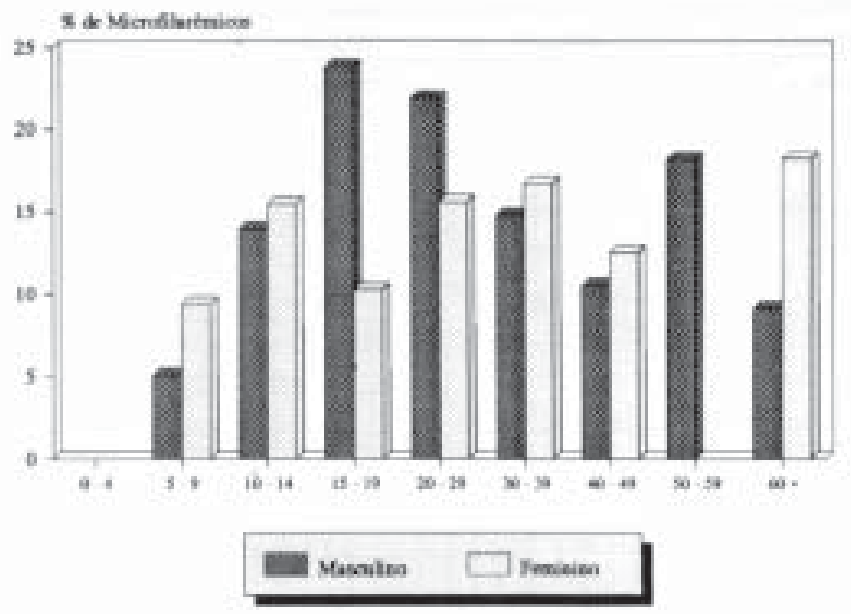

FIGURA 2. Distribuição do Índice Microfilarêmico por Sexo e Faixa Etária nos Conglomerados na Área Endêmica de Olinda, no Ano de 1991

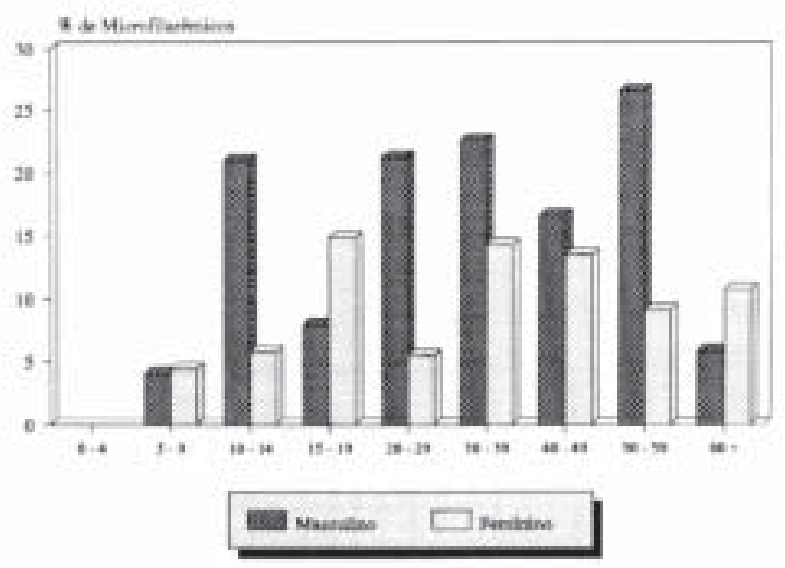

FIGURA 3. Distribuição do Índice Microfilarêmico por Faixa Etária nos Conglomerados da Áreas Endêmicas de Recife e Olinda, no Ano de 1991

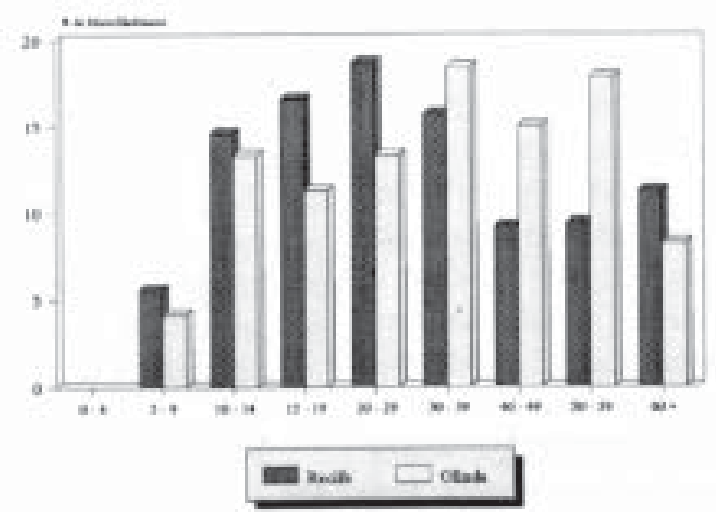


TABELA 4. Distribuição de Freqüência de Casos de Microfilarêmicos por Densidade Parasitária nos Conglomerados de Área Endêmica do Recife (Santo Amaro e Campo Grande), no Ano de 1991

\begin{tabular}{lccc}
\hline \hline Quantidade Microfilária $(60 \mu \mathrm{l})$ & Freqüência & Frequiência Cumulativa & Casos Cumulativos $(\%)$ \\
\hline 1 & 6 & 6 & 9,52 \\
2 & 4 & 10 & 15,87 \\
3 & 2 & 12 & 19,05 \\
4 & -1 & 13 & 20,63 \\
5 & - & - & 26,98 \\
6 & 4 & 17 & 30,16 \\
7 & 2 & 19 & 31,75 \\
8 & 1 & 20 & 34,92 \\
9 & 2 & 22 & 44,44 \\
10 & 6 & 28 & 53,97 \\
$11-20$ & 6 & 34 & 65,08 \\
$21-30$ & 7 & 41 & 77,78 \\
$31-40$ & 8 & 49 & 79,37 \\
$41-50$ & 1 & 50 & 80,95 \\
$51-60$ & 1 & 51 & 84,13 \\
$61-70$ & 2 & 53 & 87,30 \\
$71-80$ & 2 & 55 & 90,48 \\
$81-90$ & 2 & 57 & 95,24 \\
$91-100$ & 3 & 60 & 96,83 \\
$101-200$ & 1 & 98,41 \\
$201-300$ & 1 & 61 & 100,00 \\
$301-400$ & 1 & 62 & - \\
$401-500$ & 1 & 63 & - \\
$501-600$ & - & - & - \\
$901-900$ & - & - & - \\
\hline Total & & - & \\
\hline \hline
\end{tabular}

$-=$ Não houve casos

TABELA 5. Distribuição de Freqüência de Casos de Microfilarêmicos por Densidade Parasitária nos Conglomerados de Área Endêmica de Olinda (Salgadinho e Sapucaia), no Ano de 1991

\begin{tabular}{|c|c|c|c|}
\hline Quantidade Microfilária $(60 \mu \mathrm{l})$ & Frequiência & Freqüência Cumulativa & Casos Cumulativos (\%) \\
\hline 1 & 6 & 6 & 7,14 \\
\hline 2 & 4 & 10 & 11,90 \\
\hline 3 & 2 & 12 & 14,29 \\
\hline 4 & 1 & 13 & 15,48 \\
\hline 5 & - & - & - \\
\hline 6 & - & - & - \\
\hline 7 & 2 & 15 & 17,86 \\
\hline 8 & 2 & 17 & 20,24 \\
\hline 9 & - & & - \\
\hline 10 & 1 & 18 & 21,43 \\
\hline $11-20$ & 12 & 30 & 35,71 \\
\hline $21-30$ & 12 & 42 & 50,00 \\
\hline $31-40$ & 4 & 46 & 54,76 \\
\hline $41-50$ & 3 & 49 & 58,33 \\
\hline $51-60$ & 4 & 53 & 63,10 \\
\hline $61-70$ & 3 & 56 & 66,67 \\
\hline $71-80$ & 3 & 59 & 70,24 \\
\hline $81-90$ & 3 & 62 & 73,81 \\
\hline $91-100$ & 1 & 63 & 75,00 \\
\hline $101-200$ & 9 & 72 & 85,71 \\
\hline $201-300$ & 8 & 80 & 95,24 \\
\hline $301-400$ & 2 & 82 & 97,62 \\
\hline $401-500$ & - & - & - \\
\hline $501-600$ & - & - & - \\
\hline $601-900$ & 2 & 84 & 100,00 \\
\hline $901+$ & - & - & - \\
\hline Total & 84 & - & - \\
\hline
\end{tabular}

$-=$ Não houve casos 


\section{DISCUSSÃO}

O estudo descritivo por conglomerados das áreas do Recife e Olinda demonstrou, de maneira global, prevalências semelhantes de microfilaremia de $13,5 \%$ e $12,3 \%$, respectivamente; a densidade microfilarêmica foi mais intensa em Olinda, com valor médio de $70 \mathrm{mf} / 60 \mu \mathrm{l}$, do que em Recife, de $41 \mathrm{mf} / 60 \mu \mathrm{l}$.

Em 1952, Azevedo e Dobbin Jr. descreveram em Recife áreas consideradas de alta endemicidade, como, por exemplo, o bairro de Santo Amaro, cuja prevalência então era de $9,7 \%$.

Em relação aos índices microfilarêmicos, os picos nos bairros estudados de Recife e Olinda foram nas faixas etárias de 20-29 anos e 30-39 anos, respectivamente, apesar de a média verificada a partir dos 10 anos ter sido $14 \%$.

A distribuição por sexo e idade, das áreas comparadas, apresentou padrão semelhante ao mencionado por Sasa et al. (1976), que refere, mundialmente, nas áreas endêmicas de filariose linfática por $W$. bancrofti, que a faixa etária de 5-9 anos apresenta índices microfilarêmicos relativamente baixos, aumentando gradualmente com a idade. Nesse sentido, são também semelhantes aos encontrados no Pará (Costa, 1954), como também no Haiti (Raccurt et al., 1988), e Índia (Udonsi, 1988).

$\mathrm{Na}$ análise por qui-quadrado das variáveis sexo e microfilaremia, Olinda apresentou um predomínio do sexo masculino, enquanto, em Recife, não se verificou relação entre essas variáveis.

O sexo masculino é fator predisponente à infecção parasitária quer em Recife, quer em Olinda, sem nos permitir discussões mais aprofundadas sobre infecção/doença, pois a maioria dos estudos já realizados na Região Metropolitana do Recife é demanda de ambulatórios especializados em filariose, não envolvendo, portanto, a população em geral. $\mathrm{Na}$ Índia, em populações urbanas, a microfilaremia também está relacionada ao sexo masculino (Pani et al., 1991), enquanto, na Indonésia, há apenas moderado aumento da microfilaremia no sexo masculino, mas sem significância estatística (Dennis et al., 1976).

Acima de 60 anos,entretanto, em ambas as áreas, o sexo feminino apresentou índices mais elevados do que o masculino, o que pode ser analisado por diferentes aspectos. Nessa faixa etária, o número de mulheres examinadas foi maior do que o de homens. A esperança de vida do sexo feminino ao nascer é maior do que a do masculino, para a região metropolitana do Recife(IBGE, 1980).

É importante destacar também os fatores que afetam a transmissão da endemia, como a biologia do vetor, fatores ambientais (saneamento, habitação), fatores ralacionados ao hospedeiro, proteção individual, nível de exposição (especula-se que homens tendem a expor mais o corpo do que as mulheres), parâmetros que influenciam a prevalência, densidade parasitária e morbidade.

Em Olinda, área de ocupação mais recente, embora se desconheça a procedência e as condições da população que ali se instalou, surpreendem as taxas semelhantes de infecção que verificamos em relação a Recife, fato agravado pelo predomínio de maior densidade microfilarêmica que vem a ser responsável por maior morbidade. As verificações de maior densidade microfilarêmica em Olinda em relação a Recife corroboram as de Medeiros et al. (1992), que observaram índices mais altos de infectividade do Culex quinquefasciatus em bairros de Olinda comparados a bairros de Recife.

Em Recife, há várias décadas, existe um trabalho institucional de controle desenvolvido pela Sucam. Nessas áreas, porém, as taxas divulgadas giravam em torno de $1,5 \%$ de infectados (MS, 1985), correspondentes, portanto, à proporção de tratados. A diferença que verificamos se deve, provavelmente, tanto à metodologia aplicada em relação ao horário de coleta, em torno das $20 \mathrm{~h}$ e $24 \mathrm{~h}$, enquanto o da Sucam é de $19 \mathrm{~h}$ às $21 \mathrm{~h}$, e sabemos que a periodicidade do parasito no sangue para a nossa região varia das 23h à 01h (Dreyer \& Medeiros, 1990). Outro fator que explicaria as discrepâncias entre os achados da Sucam e os nossos é, sem dúvida, a quantidade de sangue coletada. Enquanto a Sucam coletava apenas uma gota de sangue, nossos dados se baseiam na coleta de três gotas sucessivas. Finalmente, em nosso trabalho, contamos com a participação da população. Nesse sentido, nas áreas selecionadas, houve um trabalho de esclarecimento e conscientização das comunidades sobre a endemia, sua 
transmissão e morbidade, que muito contribuiu para maior aceitação do inquérito epidemiológico.

Antes da realização das visitas domiciliares noturnas, como também durante o tratamento dos microfilarêmicos, eram realizadas palestras nas associações de bairro para explicar a importância do nosso estudo e o cumprimento do tratamento.

Pelos resultados, depreende-se que os trabalhos de controle efetuados institucionalmente devem ter contribuído para alguma redução da densidade parasitária, porém não asseguraram a redução dos níveis endêmicos nem a delimitação geográfica da endemia que, ao contrário, se estende para novas áreas. Em 1952, o bairro de Santo Amaro (Recife) apresentava 9,7\% de prevalência. Durante o período de 1960 a 1985 , os índices foram declinando até chegar a 1,5\% segundo os últimos boletins da Sucam (MS, 1985). No entanto, segundo nossos resultados, essas taxas atingem 13,5\% (1991). Diante disso, concluímos que as campanhas de controle realizadas pela Sucam devem ser reavaliadas em áreas de alta endemicidade, já que não alcançaram resultados satisfatórios.

A reflexão a respeito da relação existente entre a filariose e a desigualdade social também deve ser feita, sobretudo considerando sua nítida associação com as condições sócio-econômicas da população, já que a cadeia epidemiológica de transmissão nos bolsões de pobreza encontra condições mais favoráveis pela deficiência ou ausência de saneamento, habitação precária e desproteção ao vetor.

Os autores concluem que a área metropolitana do Recife é endêmica de filariose linfática; contudo, não se sabe com certeza qual a sua extensão, embora os índices apresentados sugiram o baixo nível de saúde em que se encontra a população, oferecendo, no que tange à pesquisa, amplas investigações no campo da biologia e do social, voltadas preferencialmente para o controle da endemia.

\section{AGRADECIMENTOS}

Agradecemos ao Setor de Informática do Centro de Pesquisas Aggeu Magalhães da Fundação Oswaldo Cruz, Dr. Wayner de Souza e George Tadeu, pela confecção das tabelas e gráficos.

\section{RESUMO}

MACIEL, M. A. V.; MARZOCHI, K. B. F.; SILVA, E. C.; ROCHA, A. \& FURTADO, A. F. Estudo Comparativo de Áreas Endêmicas de Filariose Bancroftiana na Região Metropolitana do Recife, Brasil. Cad. Saúde Públ., Rio de Janeiro, 10 (suplemento 2): 301-309, 1994.

Para se realizar o estudo comparativo da filariose bancroftiana na área metropolitana do Recife, foram selecionados dois bairros da cidade do Recife (Santo Amaro e Campo Grande) e dois bairros da cidade de Olinda (Sapucaia e Salgadinho), segundo critério apoiado na semelhança das características sócio-econômicas e demográficas e dos níveis endêmicos de filariose. As áreas foram divididas em conglomerados, com 25 casas em média. A amostra populacional foi estratificada por sexo e faixa etária de 0-4, 5-9, 10-14, 15-19, 20-29, 30-39, 40-49, 50-59 e igual ou superior a 60 anos. Os dados parasitológicos foram obtidos por meio da gota espessa mensurada ( $60 \mu$ l de sangue), coletada entre as 20 e 24 horas, processada e corada pelo método de Carrazi. Os dados obtidos foram descritos em tabelas, e a densidade parasitária e freqüência microfilarêmica analisadas através de gráficos com expressão logarítima. De maneira global, Recife apresentou prevalência de 13,5\%, e Olinda, 12,3\%. Quando comparamos a densidade parasitária, Olinda apresentou valor médio de 70 microfilárias por $60 \mu 1$ de sangue, e Recife, 41. Em relação aos índices microfilarêmicos, o maior ocorreu na faixa etária de 20-29 anos. Em Olinda, por outro lado, situou se entre 30-39 anos e em indivíduos do sexo masculino. Os autores concluem que a endemia apresenta níveis endêmicos do passado, e os dados descritos alertam para uma reavaliação das campanhas de controle realizadas pela Fundação Nacional de Saúde (FNS), já que a endemia atinge dimensões ainda não mensuradas.

Palavras-Chave: Filariose; Wuchereria bancrofti, Epidemiologia 


\section{REFERÊNCIAS BIBLIOGRÁFICAS}

AZEVEDO, R. \& DOBBIN JR., J. E., 1952. Filariose Wuchereria bancrofti no grupo residencial do IAPB no bairro dos Afogados (Recife). Publicações Avulsas do Instituto Aggeu Magalhães, 1: 157-192.

COSTA, O. R., 1954. Contribuição ao conhecimento da filariose na Amazônica. Revista do Serviço Especial de Saúde Pública, 8: 329-422.

DENNIS, D. T.; PARTONO, F.; PURMONO, P. \& SOROSO, J. P., 1976. Timor filariasis: epidemiological and clinical features in a defineded community. The American Fournal of Tropical Medicine and Hygiene, 25: 797-802.

DREYER, G., 1987. Filariasis programm Recife, Brasil. Memórias do Instituto Oswaldo Cruz, 83: 350-359.

DREYER, G. \& MEDEIROS, Z., 1990. Filariose linfática: ainda um desafio. Ciência Hoje, 12: 06-07.

IBGE (Fundação Instituto Brasileiro de Geografia e Estatística), 1982. IX Recenseamento Geral Brasileiro, 1980 (Pernambuco, Censo Demográfico. Dados Distritais). Vol. 1, T. 3, n. 10, Rio de Janeiro: IBGE.

MEDEIROS, Z.; DREYER, G.; ANDRADE, L.; PIRES, M. L.; MENDES, J. \& PIMENTEL, R., 1992. Wuchereria bancrofti, microfilarial density of autochthonous cases and natural Culex infectivity rates in northeast Brazil. The American Fournal of Tropical Medicine and Hygiene, 95: 214-217.

MS (Ministério da Saúde), 1985. O Controle das Endemias no Brasil (de 1979 a 1984). Brasília: Superitendência de Campanhas de Saúde Pública, MS. (Mimeo.)

OMS (Organização Mundial de la Salud), 1988. Lucha Contra la Filariasis Linfática - Manual para Personal Sanitario. Genebra: Organización Mundial de la Salud.

PANI, S. P.; BALAKRISNAN, N.; SRIVIDYA, A.; BUNDY, D. A. P. \& GREFELL, B. T., 1991. Clinical epidemiology of bancroftian filariasis: effect of age and gender. Transactions of the Royal Society of Tropical Medicine and Hygiene, 85: 260-264.
PARAHYM, O., 1961. Endemias Brasileiras. Recife: Universidade do Recife, Imprensa Universitária. (Coleção Nordestina)

RACCURT, P. C.; LOWRIF, C. R.; SEPHEN JR., P. K. \& YARSETH, T. D., 1988. Epidemiology of Wuchereria bancrofti in Leogane, Haiti. Transactions of the Royal Society of Tropical Medicine and Hygiene, 82: 721-725.

RACHOU, R. G., 1954. Índices epidemiológicos em inquéritos de filariose bancroftiana. Revista Brasileira de Malariologia e Doenças Tropicais, 6: $35-40$.

, 1957a. Distribuição geográfica das filarioses humanas no Brasil. Revista Brasileira de Malariologia e Doenças Tropicais, 9: 79-100. , 1957b. Considerações sobre o combate à filariose bancroftiana no Brasil. Revista Brasileira de Malariologia e Doenças Tropicais, 9: 527-536.

RACHOU, R. G. \& DEANE, L. M., 1952. Os primeiros passos do serviço nacional de malária em sua campanha contra a filariose bancroftiana no Brasil. Revista Brasileira de Malariologia e Doenças Tropicais, 4: 187-197.

RACHOU, R. G.; VILLELA, A. M.; CRUZ, A. E. \& CARVALHO, G. A., 1957. A filariose bancroftiana em Recife (Pernambuco). Resultado de um inquérito realizado em 1954. Revista Brasileira de Malariologia e Doenças Tropicais, 8: 359-367.

SASA, M.; KANDA, T.; MITSUII, G.; SHIRISAKA, A.; ISHIII, A. \& ALDCHINZEL, H., 1976. The filariasis control programs in Japan and their evaluation by means of epidemiology analysis of the microfilaria survey data. In: Recent advances in research on Filariasis in Fapan (M. Sasa, ed.), pp. 3-72, Baltimore: Park Press.

UDONSI, J. K., 1988. Bancroftian filariasis in the Ingwun Basin, Nigéria. Act Tropical, 45: 171179.

WHO (World Health Organization), 1992. Fifth Report of the WHO Expert Committee on Filariasis, 1992. Lymphatic Filariasis: The Disease and its Control. Geneva: WHO. 